Теорія Ймовір. та Матем. Статист. Вип. 78, 2008
Theor. Probability and Math. Statist.

No. 78, 2009, Pages 61-73

S 0094-9000(09)00762-5

Article electronically published on August 4, 2009

\title{
INHOMOGENEOUS PERTURBATIONS OF A RENEWAL EQUATION AND THE CRAMÉR-LUNDBERG THEOREM FOR A RISK PROCESS WITH VARIABLE PREMIUM RATES
}

\author{
UDC 519.21
}

\author{
M. V. KARTASHOV
}

\begin{abstract}
We consider a time inhomogeneous perturbation of the classical renewal equation with continuous time that can be reduced to the integral Volterra equation with a nonnegative bounded kernel. We assume that the kernel is approximated for large time intervals by a convolution kernel generated by a probability distribution. We prove that the limit of the solution of the perturbed equation exists if the corresponding perturbation of solutions of the perturbed equation is small.

We consider an application for ruin functions of the classical risk process where the premium rate depends on the current capital of an insurance company. We obtain the exponential asymptotic behavior with the Lundberg index evaluated from the original (nonperturbed) intensity.
\end{abstract}

\section{INTRODUCTION}

The risk processes in an inhomogeneous environment are studied in the papers [2]-[16].

We consider a time inhomogeneous generalization of the classical renewal equation with continuous time that can be reduced to the integral Volterra equation with a nonnegative bounded kernel. The main assumption is that, for large time intervals, the kernel is approximated in variation by a convolution kernel generated by a probability distribution.

The setting of the problem arose from the studies of the asymptotic behavior of the ruin function for a risk process with variable premium rates considered in [16]. We essentially improve the conditions imposed on the asymptotic behavior of the perturbation; namely, we assume the linear behavior of the perturbation in contrast to [16] where the behavior is exponential.

The proofs in the first part of the paper use the ideas of an unpublished author, paper [17] and those of Schmidli [13. It is assumed in [13] that the perturbed kernel is subordinate to the limit convolution kernel. Another assumption in [13] is the direct Riemann integrability. The results proved below hold without these two assumptions.

We prove that the limit at infinity of a solution of the perturbed equation exists if the corresponding perturbation is small in a certain sense.

In the second part of the paper, we show some applications by obtaining the asymptotic behavior of the ruin function for the classical ruin process where the premium rate depends on the current capital of an insurance company. We prove that the risk function is exponential with the Lundberg index evaluated from the limit premium rate. This

2000 Mathematics Subject Classification. Primary 60J45; Secondary 60A05, 60K05.

Key words and phrases. Volterra equation, renewal theorem, risk function, Lundberg index, Poisson process. 
generalization of the Lundberg-Cramér theorem is proved under a minimal assumption that the difference between the premium rate and the limit rate is integrable at infinity.

\section{Perturbed Renewal equation}

Let $G$ be a probability measure on $\mathbb{R}=(-\infty, \infty)$.

The integral equation

$$
x(t)=y(t)+\int_{\mathbb{R}} x(t-s) G(d s), \quad t \in \mathbb{R},
$$

with an unknown function $x$ considered in the class

$$
B_{0}(\mathbb{R}) \equiv\left\{x: \mathbb{R} \rightarrow \mathbb{R}, x \text { is a Borel function such that } \sup _{s \leq t}|x(s)|<\infty \text { for all } t \geq 0\right\}
$$

is called the renewal equation on $\mathbb{R}$ generated by the measure $G$.

We also consider the following class of functions:

$$
L_{1}^{0}(\mathbb{R}) \equiv\left\{y \in B_{0}(\mathbb{R}): \lim _{t \rightarrow \infty} y(t)=0, \int_{\mathbb{R}}|y(s)| d s<\infty\right\} .
$$

Recall that [19] a measure $G$ is of the absolutely continuous type if the convolution $G^{* m}$ has an absolutely continuous component for some $m \geq 1$, that is, if

$$
G^{* m}(B) \geq \int_{B} h(s) d s
$$

for all Borel sets $B$ and some nonnegative function $h$ that does not vanish almost everywhere.

One of the consequences of the nonuniform case of Theorem 1 in 19 (namely, the implication $(K, M) \Longrightarrow(R))$ implies the following result if $G\left(\mathbb{R}_{+}\right)=1$.

Renewal Theorem for distributions of the absolutely continuous type. Let a probability distribution $G$ be of the absolutely continuous type and have a finite mean value

$$
m_{G} \equiv \int_{\mathbb{R}} s G(d s) \in(0, \infty) .
$$

Then, for every function $y \in L_{1}^{0}(\mathbb{R})$, equation (1) has a unique solution $x$ in the class $B_{0}(\mathbb{R})$. Moreover, the limit

$$
\lim _{t \rightarrow \infty} x(t)=m_{G}^{-1} \int_{\mathbb{R}} y(s) d s
$$

exists. In particular, $\sup _{t \in \mathbb{R}}|x(t)|<\infty$.

This result can be proved for the case of $G\left(\mathbb{R}_{+}\right)<1$ by applying the method of ladder heights [20, Chapter XII.3]. See [23, Appendix 2, Theorem 6] or [24, 25.

Definition. The generalized integral Volterra equation

$$
x(t)=y(t)+\int_{\mathbb{R}} x(t-s) F(t, d s), \quad t \in \mathbb{R},
$$

for the function $x \in B_{0}(\mathbb{R})$ is called an inhomogeneous perturbation of a renewal equation (11) if the kernel $F$ is measurable, nonnegative, and asymptotically approaches the probability distribution $G$ in variation, that is,

$$
\Delta(t) \equiv \int_{\mathbb{R}}|\delta|(t, d s) \rightarrow 0, \quad t \rightarrow \infty,
$$


where the perturbation $\delta$ of the kernel $G$ is equal to

$$
\delta(t, d s) \equiv F(t, d s)-G(d s) .
$$

The following result requires a restriction on the boundedness of a solution of equation (4).

Theorem 1. Let a probability distribution $G$ be of the absolutely continuous type and have a finite mean value (2). Assume that

(1) $y \in L_{1}^{0}(\mathbb{R})$,

(2) the averaged perturbation $\Delta$ defined by (5) is such that $(\Delta(t), t \in \mathbb{R}) \in L_{1}^{0}(\mathbb{R})$,

(3) the solution $x \in B_{0}(\mathbb{R})$ of equation (4) is bounded.

Then the limit $\lim _{t \rightarrow \infty} x(t)$ exists and is finite.

The uniqueness of a solution of equation (4) follows from the following result.

Theorem 2. Let one of the following conditions hold:

(a) there exists the density $f_{t}(s)$ of the measure $F(t, d s)$ bounded in $t$ and $s$ and moreover $F(t, \overline{[0, t]})=0$ for all $t>0$,

(b) $F\left(t, \mathbb{R}_{-}\right)=0$ and $\sup _{t<T} F(t, \mathbb{R})<1$ for all $T>0$,

(c) the measure $G\left(B \cap \mathbb{R}_{+}\right)$is of the absolutely continuous type and satisfies condition (21); the perturbation $\Delta$ defined by (51) is such that $(\Delta(t), t \in \mathbb{R}) \in L_{1}^{0}(\mathbb{R})$; the following norm

$$
\|\Delta\|_{01} \equiv \sup _{t \in \mathbb{R}} \Delta(t)+\int_{\mathbb{R}} \Delta(t) d t
$$

is sufficiently small.

Then equation (4) has a unique solution $x$ in the class $B_{0}(\mathbb{R})$ for every $y \in L_{1}^{0}(\mathbb{R})$. Moreover, the solution coincides with the sum of the series

$$
x(t)=\sum_{n \geq 0} F^{n}[y](t)
$$

that uniformly converges on finite intervals. Here $F^{n}$ is the $n$-fold product of the bounded integral operator $F$ acting on $B_{0}(\mathbb{R})$ as follows:

$$
F[y](t) \equiv \int_{\mathbb{R}} y(t-s) F(t, d s), \quad t \in \mathbb{R} .
$$

Remark. If $y \in B_{0}(\mathbb{R})$ is nonnegative and series (8) converges, then its sum is the minimal solution of equation (4).

The boundedness of the solution $x$ follows if the kernel is substochastic and the rate of convergence of the perturbation to zero is sufficiently high.

Theorem 3. Assume that the probability distribution $G$ is of the absolutely continuous type and has the finite mean value (2). Let $x \in B_{0}(\mathbb{R})$ be a solution of the inhomogeneous renewal equation (4) represented by the sum of absolutely convergent series (8). Let the following conditions hold:

(1) $y \in L_{1}^{0}(\mathbb{R})$,

(2) the perturbation $\delta$ defined by (6) is such that $\left(\Delta_{1}(t), t \in \mathbb{R}\right) \in L_{1}^{0}(\mathbb{R})$, where the weighted perturbation $\Delta_{1}$ is given by

$$
\Delta_{1}(t)=\int_{\mathbb{R}}\left(1+t^{+}+|s|\right)|\delta|(t, d s),
$$

(3) $\sup _{t \leq T} F(t, \mathbb{R})<1$ for all $T>0$. 
Then the limit $\lim _{t \rightarrow \infty} x(t)$ exists and is finite.

Further, if $\int_{0}^{\infty} y(s) d s \neq 0$ and

(a) $G(T)<1$ for all $T>0$,

(b) the norm $\|\Delta\|_{01}$ (see (7)) of the weighted perturbation $\Delta_{1}(t)$ defined by (10) is sufficiently small,

then the limit $\lim _{t \rightarrow \infty} x(t)$ is nonzero.

Remark. All the restrictions imposed on $x$ in the first part of Theorem 3 hold under the assumptions of Theorem 2. Conditions (a) and (b) of the second part of the theorem imply condition (3).

\section{INHOMOGENEOUS RISK PROCESSES}

Let $c(x), x \in \mathbb{R}_{+}$, be a Borel function bounded and separated from zero, that is,

$$
0<\inf _{x \geq 0} c(x), \quad \sup _{x \geq 0} c(x)<\infty .
$$

Define the following compound Poisson process:

$$
Z(t)=\sum_{k=1}^{\nu(t)} \xi_{k},
$$

where $\left(\xi_{n}, n \geq 1\right)$ are independent nonnegative identically distributed insurance claims that are independent of the Poisson process $\nu(t)$.

Consider a right continuous Markov process determined by the following stochastic equation:

$$
d X_{t}=c\left(X_{t}\right) d t-d Z_{t}, \quad t \geq 0, X_{0}=x .
$$

The ruin probability is defined as

$$
q(x)=\mathrm{P}\left(\bigcup_{t \geq 0}\left\{X_{t}<0\right\} \mid X_{0}=x\right), \quad x \geq 0 .
$$

Denote

$$
\lambda=\mathrm{E} \nu(1), \quad m=\mathrm{E} \xi_{1}, \quad F(x)=\mathrm{P}\left(\xi_{1}<x\right), \quad \bar{F}(x)=1-F(x) .
$$

Let the Cramér condition hold:

$$
\gamma \equiv \sup \left(s \geq 0: \operatorname{E} \exp \left(s \xi_{1}\right)<\infty\right)>0 .
$$

If condition (15) holds for $\operatorname{Re} s<\gamma$, then the moment generating function

$$
\widehat{f}(s)=\int_{0}^{\infty} \exp (s x) d F(x)=\mathrm{E} \exp \left(s \xi_{1}\right)
$$

is well defined and analytic.

Let the premium rate function have the limit $c>0$ at infinity satisfying the balance equation

$$
\lim _{x \rightarrow \infty} c(x)=c>\lambda m .
$$

Consider the Lundberg index for the risk problem with the constant premium rate $c$ :

$$
\alpha \equiv \sup (s<\gamma: \lambda \widehat{f}(s)-\lambda<c s) \in(0, \infty) .
$$

The positivity of $\alpha$ is a consequence of (17) and (15), while the boundedness of $\alpha$ follows from the representation

$$
s=o(\widehat{f}(s)), \quad s \rightarrow \infty .
$$


Since the function $\widehat{f}(s)$ is left continuous and convex in $[0, \gamma]$, we derive from the Lundberg condition

$$
\widehat{f}^{\prime}(\alpha)<\infty, \quad \bar{F}(t)=o(\exp (-\alpha t)), \quad t \rightarrow \infty,
$$

that the number $\alpha=\alpha(c)$ is a unique positive solution of the equation

$$
\lambda \widehat{f}(\alpha)-\lambda-c \alpha=0 .
$$

Notice that (19) always holds if $\alpha<\gamma$.

Theorem 4. Let a Markov process $\left(X_{t}, t \geq 0\right)$ be defined by equation (12). Assume that Cramér's condition (15) and the boundedness condition (11) hold. We also assume that the balance condition (17) and Lundberg's condition (19) are satisfied for some constant $c>0$, where the numbers $\alpha \leq \gamma$ are defined by (18) and (15), respectively.

If $(c(x)-c, x \geq 0) \in L_{1}^{0}\left(\mathbb{R}_{+}\right)$, then the limits

$$
\lim _{x \rightarrow \infty} \exp (\alpha x) q^{\prime}(x), \quad \lim _{x \rightarrow \infty} \exp (\alpha x) q(x)
$$

exist and are finite.

Further, if the perturbation $c(x)-c$ is sufficiently small and $F(T)<1$ for all $T>0$, then the limits (21) are nonzero.

Example. Let the premium rate $c(x)$ satisfy condition (11) and let the limit

$$
\lim _{x \rightarrow \infty} c(x)=c>0
$$

exist. Assume that the claims are exponentially distributed, that is, $\bar{F}(x)=\exp (-x)$, $x \geq 0$. The balance condition (17) becomes of the form $\beta \equiv \lambda / c<1$ in this case. Then

$$
q^{\prime}(x)=-\gamma \frac{c}{c(x)} \exp \left(-\alpha x-\beta \int_{0}^{x} \frac{c(s)-c}{c(s)} d s\right),
$$

where $\alpha=1-\beta$ and $\gamma=\beta(1-q(0))$. The value $q(0)$ is evaluated after the integration of representation (22) on $\mathbb{R}_{+}$, since the integral on $\mathbb{R}_{+}$of the left hand side is equal to $-q(0)$.

Note that the second term in the exponent of (22) is $o(x)$ as $x \rightarrow \infty$. Thus $\alpha$ is the exact index of the exponential asymptotics for the derivative of the risk function $q^{\prime}(x)$.

We derive the following three results concerning the necessity of the main assumption of Theorem 3 from representation (22).

Proposition (a). Assume that the perturbation $c(x)-c$ does not change its sign. In order that the limit $\lim _{x \rightarrow \infty} \exp (\alpha x) q(x)$ exist and be finite and nonzero it is necessary and sufficient that $(c(x)-c, x \geq 0) \in L_{1}^{0}\left(\mathbb{R}_{+}\right)$.

Proposition (b). The limit $\lim _{t \rightarrow \infty} \exp (\alpha x) q^{\prime}(x)$ exists and is finite and nonzero if and only if the improper integral

$$
I(\infty) \equiv \lim _{x \rightarrow \infty} \int_{0}^{x}(c(s)-c) c^{-1}(s) d s
$$

is finite.

Proposition (c). If the integral $I(\infty)$ is finite, then

$$
\lim _{x \rightarrow \infty} \exp (\alpha x) q(x)=\gamma \alpha^{-1} \exp (-\beta I(\infty)) .
$$




\section{ThE PROOF OF GENERALIZED RENEWAL THEOREMS}

Recall that the perturbation kernel $\delta$ is defined by relation (6).

Proof of Theorem 1. We rewrite (4) according to (6) in the form of a renewal equation, namely

$$
x(t)=y_{1}(t)+\int_{\mathbb{R}} x(t-s) G(d s), \quad t \in \mathbb{R},
$$

where the function

$$
y_{1}(t) \equiv y(t)+\int_{\mathbb{R}} x(t-s) \delta(t, d s)
$$

is estimated by (5) as follows:

$$
\left|y_{1}(t)\right| \leq|y(t)|+\Delta(t) \sup _{s \in \mathbb{R}}|x(s)| .
$$

We derive from the assumptions of the theorem that $y_{1} \in L_{1}^{0}(\mathbb{R})$.

Applying the above Renewal Theorem for distributions of the absolutely continuous type to equation (24) we complete the proof.

Proof of Theorem 2. Applying the successive iterations we obtain the following equation from (4):

$$
x(t)=\sum_{0 \leq k<n} F^{k}[y](t)+F^{n}[x](t) .
$$

(a) Theorem 2 follows, since the integration in (4) is over the interval $[0, t]$ for $t>0$, that is, $F$ is a Volterra operator on $B_{0}(\mathbb{R})$ :

$$
\sup _{t \leq T}\left|F^{n}[y](t)\right| \leq\left(\sup _{0 \leq s \leq t \leq T} f_{t}(s)\right)^{n} \sup _{s \leq T}|y(s)| / n ! \rightarrow 0, \quad n \rightarrow \infty .
$$

(b) The operator $F[\cdot]$ in (9) is contractive and acting in the space of bounded Borel functions defined on $(-\infty, T]$ :

$$
\sup _{t \leq T}|F[y](t)|=\sup _{t \leq T}\left|\int_{\mathbb{R}_{+}} y(t-s) F(t, d s)\right| \leq \sup _{t \leq T} F(t, \mathbb{R}) \sup _{t \leq T}|y(t)| .
$$

(c) Note that the class $L_{1}^{0}(\mathbb{R})$ is closed with respect to the convolution with a finite measure.

Denote by $\delta[x]$ the integral operator of the form (9) which is generated by the perturbation kernel (6).

Equation (4) is equivalent to

$$
x=y+\delta[x]+x * G .
$$

Since $\delta[x] \in B_{0}(\mathbb{R})$, equation (27) is equivalent to

$$
x=U * y+U * \delta[x],
$$

where $U=\sum_{n>0} G^{n}$ is the renewal measure. Using the Stone representation [24, 25] and [19, Theorem 1 , the implication $(K, M) \Longrightarrow(S)$ ] we represent the measure $U$ as the sum $U=V+H$, where $V$ is a finite measure and the measure $H$ has a bounded density. Since $y \in L_{1}^{0}(\mathbb{R})$, the function $U * y$ is bounded. If the norm (17) is sufficiently small, then the total variation of the kernel $U * \delta(x, B)$ is less than $1 / 2$ uniformly in $x$. Considering the uniformly convergent Neumann series for the solution of equation (28) we prove that the solution exists and is unique in the class $B_{0}\left(\mathbb{R}_{+}\right)$. The above transformations are equivalent, whence we derive the corresponding statement for equation (4). 
If $y \in L_{1}^{0}(\mathbb{R})$ is nonnegative, the monotone limit as $n \rightarrow \infty$ in (26) is a solution of (44), since

$$
F[x]=F\left[\sum_{0 \leq k<\infty} F^{k}[y]\right]=\sum_{0 \leq k<\infty} F^{k+1}[y]=x-y
$$

by the monotone convergence theorem. Thus the limit of the second term on the right hand side of (26) equals zero by the uniqueness proved above. This proves (8).

Comparison Lemma. Let $x, \bar{x} \in B_{0}(\mathbb{R})$ be solutions of equation (4) corresponding to the functions $y, \bar{y} \in B_{0}(\mathbb{R})$ and let $x$ and $\bar{x}$ equal the sums of the corresponding absolutely convergent series (8).

If $y(t) \leq \bar{y}(t)$ for all $t$, then $x(t) \leq \bar{x}(t), t \in \mathbb{R}$.

This result follows from the comparison theorem for absolutely convergent series, since the kernel $F$ is nonnegative, whence $F^{n}[y] \leq F^{n}[\bar{y}]$.

Proof of Theorem 3. Let $x \in B_{0}(\mathbb{R})$ be a solution of equation (4) represented in the form (8) for $y \in L_{1}^{0}(\mathbb{R})$. Put $c \equiv \sup _{t \in \mathbb{R}}|y(t)|<\infty$.

Consider the function $\bar{x}(t)=a+b t^{+}$for some $a, b>0$. This function satisfies equation (4) with the following right hand side:

$$
\begin{aligned}
\bar{y}(t) & =a+b t^{+}-\int_{\mathbb{R}}\left(a+b(t-s)^{+}\right) F(t, d s) \\
& =\left(a+b t^{+}\right)(1-F(t, \mathbb{R}))+b \int_{\mathbb{R}}\left(t^{+}-(t-s)^{+}\right) F(t, d s) .
\end{aligned}
$$

Since $\left|t^{+}-(t-s)^{+}\right| \leq|s|$ for $t \geq 0$, we have

$$
\left|\int_{\mathbb{R}}\left(t^{+}-(t-s)^{+}\right) \delta(t, d s)\right| \leq \int_{\mathbb{R}}|s||\delta|(t, d s) \rightarrow 0, \quad t \rightarrow \infty,
$$

because (10) is a member of the class $L_{1}^{0}(\mathbb{R})$.

By definition (6) and condition (2),

$$
\begin{aligned}
& \underline{\lim _{t \rightarrow \infty}} \int_{\mathbb{R}}\left(t^{+}-(t-s)^{+}\right) F(t, d s) \\
& \quad \geq \varliminf_{t \rightarrow \infty} \int_{\mathbb{R}}\left(t^{+}-(t-s)^{+}\right) G(d s)-\varlimsup_{t \rightarrow \infty}\left|\int_{\mathbb{R}}\left(t^{+}-(t-s)^{+}\right) \delta(t, d s)\right| \\
& \quad=\int_{\mathbb{R}} s G(d s)>0 .
\end{aligned}
$$

The first term on the right hand side of (29) is positive in view of assumption (3) of the theorem. Thus (30) and the inclusion $y \in L_{1}^{0}(\mathbb{R})$ imply that the right hand side of (29) is not less than $c$ for $t \geq T$ uniformly for all $a>0$ where $T>0$ and $b>0$ are some numbers. Taking into account assumption (3) of the theorem and the following upper bound,

$$
\begin{aligned}
& \varlimsup_{t \rightarrow-\infty}\left|\int_{\mathbb{R}}\left(t^{+}-(t-s)^{+}\right) F(t, d s)\right| \\
& \quad \leq \varlimsup_{t \rightarrow-\infty}\left|\int_{\mathbb{R}}(t-s)^{+} G(d s)\right|+\varlimsup_{t \rightarrow-\infty}\left|\int_{\mathbb{R}}(t-s)^{+} \delta(t, d s)\right| \\
& \quad \leq \varlimsup_{t \rightarrow-\infty}\left|\int_{(-\infty, t)}\right| s|| \delta|(t, d s)|<\infty,
\end{aligned}
$$

the right hand side of equality (29) is not less than $c$ for $t<T$ if $a$ is sufficiently large.

Thus $\bar{y}(t) \geq c \geq y(t)$ for all $t \in \mathbb{R}$ and some $a, b>0$. 
Note that $\bar{y}$ is nonnegative. Thus the series $\sum_{n>0} F^{n}[\bar{y}]$ is absolutely convergent, since its partial sums do not decrease and are bounded and since the function $\bar{x}(t)$ is bounded:

$$
\sum_{0 \leq k<n} F^{n}[\bar{y}](t)=\bar{x}(t)-F^{n}[\bar{x}](t) \leq \bar{x}(t) .
$$

Thus $\bar{x}(t) \geq x(t), t \in \mathbb{R}$, by the Comparison Lemma, that is, $x(t) \leq a+b t^{+}$for all $t \in \mathbb{R}$.

Reasoning in the same way but with $-\bar{x}$ instead of $\bar{x}$, we prove the inequality

$$
-x(t) \leq a+b t^{+}
$$

for all $t$.

Consider equation (24) for $x$. By definition (25),

$$
\begin{aligned}
\left|y_{1}(t)\right| & \leq|y(t)|+\int_{\mathbb{R}}|x(t-s)||\delta|(t, d s) d s \\
& \leq|y(t)|+\int_{\mathbb{R}}\left(a+b(t-s)^{+}\right)|\delta|(t, d s) \leq|y(t)|+(1+a+b) \Delta_{1}(t) .
\end{aligned}
$$

According to the assumptions of the theorem, the right hand side of (31) belongs to the class $L_{1}^{0}(\mathbb{R})$.

Applying the Renewal Theorem for distributions of the absolutely continuous type we

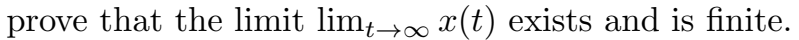

Notice that the last statement of the theorem implies condition (3) of the theorem for small perturbations $\Delta$ :

$$
\sup _{t \leq T} F(t, \mathbb{R}) \leq G(T)+\sup _{t \leq T} \Delta(t)<1 .
$$

Equality (3) of the Renewal Theorem for distributions of the absolutely continuous type and (24) yield

$$
\left|\lim _{t \rightarrow \infty} x(t)\right|=m_{G}^{-1}\left|\int_{\mathbb{R}} y_{1}(t) d t\right| \geq m_{G}^{-1}\left|\int_{\mathbb{R}} y(t) d t\right|-m_{G}^{-1}(1+a+b) \int_{\mathbb{R}} \Delta_{1}(t) d t,
$$

where we used definition (25) and a lower bound for $y_{1}$ that is analogous to (31). The reasoning concerning (29) proves also that the constant $a$ is a nonincreasing functional of $\sup _{t \in \mathbb{R}} \Delta_{1}(t)$. Thus one can assume that $a$ does not depend on the perturbation $\delta$ in a certain uniform neighborhood of zero.

The first term on the right hand side of (32) is positive by the assumption of the theorem. Therefore if $\Delta$ is sufficiently small, then the right hand side is positive (that is, nonzero).

\section{Proof of generalized Cramér-Lundberg's Theorem}

Below we recall some necessary results for the inhomogeneous risk processes proved in [16. Note that the proofs do not use the assumption on the exponential asymptotic behavior of $c(x)-c, x \rightarrow \infty$.

Lemma 2 ([16]). The ruin function $q(x)$ is nondecreasing and $q(x) \rightarrow 0$ as $x \rightarrow \infty$.

Lemma 3 ([16]). For almost all $x \geq 0$, the function $q(x)$ is differentiable and such that

$$
-c(x) q^{\prime}(x)=-\lambda q(x)+\lambda \int_{0}^{x} q(x-y) d F(y)+\lambda \bar{F}(x) .
$$

Consider the function

$$
r(x) \equiv-q^{\prime}(x) \geq 0
$$


Lemma 4 (16]). The function $r(x)$ is a solution of the equation

$$
c(x) r(x)=\lambda \int_{0}^{x} r(x-y) \bar{F}(y) d y+\lambda(1-q(0)) \bar{F}(x), \quad x \geq 0,
$$

and is such that

$$
\int_{0}^{\infty} c(x) r(x) d x=\lambda m .
$$

Note that equation (34) differs from the result of [16] by the notation: the variable $y$ is used in [16] instead of $x-y$ and $G$ stands for $\bar{F}$ in the integral (34).

Proof of Theorem 4. Let $\alpha$ be the Lundberg index as defined in (18). For $t \geq s \geq 0$, we define the following Borel functions and measures:

$$
\begin{gathered}
x(t)=\exp (\alpha t) r(t), \\
y(t)=\lambda(1-q(0)) \bar{F}(t) \exp (\alpha t) / c(t), \\
G(d s)=\lambda \bar{F}(s) \exp (\alpha s) c^{-1} 1_{s \geq 0} d s, \\
F(t, d s)=c^{-1}(t) c 1_{0 \leq s \leq t} G(d s) .
\end{gathered}
$$

We extend the domain of the functions by assuming that they are zero in $(-\infty, 0)$.

Changing $x$ and $y$ for $t$ and $s$ in (34) and multiplying the result by $\exp (\alpha t) / c$ we prove that the functions $x$ and $y$ and the measure $G$ are such that

$$
x(t) c(t) / c=y(t) c(t) / c+\int_{0}^{t} x(t-s) G(d s) .
$$

Dividing (37) by $c(t) / c$ we show that the functions $x$ and $y$ and the kernel $F$ satisfy the perturbed renewal equation (4):

$$
x(t)=y(t)+F[x](t), \quad t \geq 0 .
$$

To prove that the limit

$$
\lim _{t \rightarrow \infty} \exp (\alpha t) q^{\prime}(t) \equiv-\lim _{t \rightarrow \infty} x(t)
$$

exists we use Theorem 1.

The boundedness condition (11) and equation (34) imply that $x$ is a Borel function and $x$ is bounded on finite intervals, since $r(t)=-q^{\prime}(t)$ is integrable and the function $\bar{F}$ defined in (14) is nonincreasing.

Note that (34) implies condition (a) of Theorem 2, whence we obtain (8).

The inclusion $y \in L_{1}^{0}(\mathbb{R})$ follows from the boundedness (11) and Lundberg's condition (19), whence we conclude that $\widehat{f}(\alpha)<\infty$ and

$$
\begin{gathered}
\int_{0}^{\infty} \bar{F}(t) \exp (\alpha t) c^{-1}(t) d t \leq\left(\inf _{t \geq 0} c(t)\right)^{-1}(\widehat{f}(\alpha)-1) / \alpha<\infty, \\
\bar{F}(t) \exp (\alpha t) c^{-1}(t) \rightarrow 0, \quad t \rightarrow \infty .
\end{gathered}
$$

We derive from (20) and definition (16) that

$$
\int_{0}^{\infty} G(d s)=\lambda c^{-1} \int_{0}^{\infty} \bar{F}(s) \exp (\alpha s) d s=\lambda c^{-1} \alpha^{-1}(\widehat{f}(\alpha)-1)=1 .
$$

Therefore $G$ is a probability measure. It follows from (19) that the mean (2) is finite and equals $\lambda c^{-1} \widehat{f}^{\prime}(\alpha)$. 
The averaged perturbation (5) is given by

$$
\Delta(t)=\int_{0}^{t}|c / c(t)-1| G(d s) \leq|c(t)-c| / \inf _{s \geq 0} c(s)
$$

for $t \geq 0$. Thus $(\Delta(t), t \geq 0) \in L_{1}^{0}\left(\mathbb{R}_{+}\right)$by the main assumption of the theorem.

It remains to check the condition that the solution $x$ is bounded, and then we are ready to apply Theorem 1 .

We rewrite equation (37) in the form of the renewal equation

$$
x(t)=y(t) c(t) / c+x(t)(1-c(t) / c)+\int_{0}^{t} x(t-s) G(d s) .
$$

Denote by $U$ the renewal measure corresponding to the renewal equation, where the distribution of the regeneration time is $G$. Since $x$ and the function on the right hand side of (41) belong to the class $B_{0}(\mathbb{R})$, the unique solution of (41) in the class $B_{0}(\mathbb{R})$ can be represented [20] as the convolution of the above function and $U$; namely,

$$
x(t)=y_{0}(t)+\int_{0}^{t} x(t-s)(1-c(t-s) / c) U(d s),
$$

where

$$
y_{0}(t)=\int_{0}^{t} y(t-s)(c(t-s) / c) U(d s) .
$$

The function $y_{0}$ on the right hand side of (42) is a solution of the renewal equation for the distribution function $G$; the function $y(t) c(t) / c$ belongs to the class $L_{1}^{0}(\mathbb{R})$ in this case, since $y \in L_{1}^{0}(\mathbb{R})$ and in view of conditions (11). According to the Renewal Theorem for distributions of the absolutely continuous type the solution is bounded, that is,

$$
\sup _{t \geq 0}\left|y_{0}(t)\right|<\infty .
$$

By Stone's representation [24, 25] (also see the implication $(K, M) \Longrightarrow(S)$ in [19, Theorem 1]), the renewal measure $U$ for the absolutely continuous distribution $G$ can be represented as follows:

$$
U(B)=\delta_{0}(B)+\int_{B} h(t) d t,
$$

where $\delta_{0}$ is the Dirac measure concentrated at zero and where the renewal density $h(t) \geq 0$ has the limit value as $t \rightarrow \infty$ and is bounded, that is, $\sup _{t \geq 0} h(t)<\infty$, since $G$ possesses the density $g \in B_{0}(\mathbb{R})$.

By the above condition, the function $e(t) \equiv 1-c(t) / c$ belongs to the class $L_{1}^{0}(\mathbb{R})$. We derive the equation

$$
x(t)=y_{0}(t)+x(t) e(t)+\int_{0}^{t} x(s) e(s) h(t-s) d s
$$

from equation (42) and representation (43).

Moving the term $x(t) e(t)$ to the left hand side and then dividing both sides by $1-e(t)$ we obtain the following equation:

$$
x(t)=y_{0}(t) c / c(t)+c / c(t) \int_{0}^{t} x(s) e(s) h(t-s) d s .
$$

Put

$$
\begin{array}{ll}
\alpha(t)=|x(t)|, \quad b=\sup _{t \geq 0}\left|y_{0}(t)\right| c / c(t), \\
\varepsilon(t)=|e(t)|, \quad h=\sup _{t \geq 0}|h(t)| c / c(t) .
\end{array}
$$


Then it follows from (45) that

$$
\alpha(t) \leq b+h \int_{0}^{t} \alpha(s) \varepsilon(s) d s .
$$

Since $\alpha \in B_{0}(\mathbb{R})$ and the right hand side of (47) is a nonnegative integral Volterra operator, we follow the idea of the proof of the Comparison Lemma and conclude from (47) that $\alpha$ does not exceed the solution of the equation obtained from (47) by changing the inequality by an equality there, namely

$$
\alpha(t) \leq b+\int_{0}^{t} b h \varepsilon(s) \exp \left(\int_{s}^{t} h \varepsilon(u) d u\right) d s .
$$

By definition, $\varepsilon=|e| \in L_{1}^{0}(\mathbb{R})$. Thus inequality (48) implies the bound

$$
\sup _{t \geq 0}|x(t)| \leq b+b h \int_{0}^{\infty} \varepsilon(s) d s \exp \left(h \int_{0}^{\infty} \varepsilon(u) d u\right)<\infty .
$$

Therefore the solution $x$ of the perturbed renewal equation (38) is bounded and, moreover, all the assumptions of Theorem 1 hold.

Thus the limit (39) exists and is finite. Since

$$
\exp (\alpha t) q(t)=q(t) / \exp (-\alpha t)=\int_{t}^{\infty} r(s) d s / \exp (-\alpha t),
$$

the l'Hospital rule proves that the limit as $t \rightarrow \infty$ of the left hand side of (49) exists and is finite.

It remains to prove the last statement of the theorem. Note that the function $y$ involved in (38) and (36) is positive, whence

$$
\int_{0}^{\infty} y(t) d t>0
$$

Moreover, the condition $F(T)<1$ of Theorem 4 yields

$$
G(T)=1-\int_{T}^{\infty} G(d s)=1-\lambda c^{-1} \int_{T}^{\infty} \bar{F}(s) \exp (\alpha s) d s<1
$$

for all $T>0$.

Hence all the assumptions of the last statement of Theorem 3 hold.

Also, bound (40) implies that the perturbation $\Delta$ is small.

Therefore the last statement of Theorem 4 follows from the last statement of Theorem 3 , indeed.

Proofs for Example. Dividing (34) by $c$, substituting $\bar{F}$, changing the variables $x$ and $y$ for $t$ and $t-s$, and multiplying both sides by $\exp (\alpha t)$ we obtain the following integral equation for the normalized derivative $x(t) \equiv \exp (\alpha t) r(t)$ :

$$
x(t) c(t) / c=\beta \int_{0}^{t} x(s) d s+\gamma .
$$

Introducing the integrating factor

$$
\exp \left(-\beta \int_{0}^{t}(c / c(s)) d s\right),
$$

we evaluate (22) where the variable $t$ is changed for $x$.

(a) Suppose the perturbation $c(x)-c$ does not change its sign. Integrating over $[x, \infty)$ we deduce from (22) that

$$
\exp (\alpha x) q(x)=\gamma \int_{0}^{\infty} \frac{c}{c(s+x)} \exp \left(-\alpha s-\beta \int_{0}^{s+x} \frac{c(u)-c}{c(u)} d u\right) d s .
$$


The first factor in the integral is bounded, separated from zero, and approaches 1 as $x \rightarrow \infty$. The second term in the exponent is a monotone function. Thus one can interchange the signs of the limit as $x \rightarrow \infty$ and the integral. This means that the limit (23) is incorporated in the exponent for the integral. Therefore this limit is finite if and only if the limit on the left hand side of (50) is finite and positive.

(b) This case is an obvious corollary of (22), since

$$
\lim _{x \rightarrow \infty} c(x)=c,
$$

that is,

$$
\lim _{x \rightarrow \infty} \exp (\alpha x) r(x)=\gamma \exp (-\beta I(\infty)) .
$$

(c) This case follows from case (b) by applying the l'Hospital rule to (49).

\section{BIBLIOGRAPHY}

1. N. V. Kartashov, Strong Stable Markov Chains, VSP/TViMS, Utrecht/Kiev, The Netherlands/Ukraine, 1996. MR/1451375 (99e:60150)

2. S. Asmussen and S. S. Petersen, Ruin probabilities expressed in terms of storage processes, Adv. Appl. Probab. 20 (1988), 913-916. MR968005 (90f:62312)

3. S. Asmussen and H. M. Nielsen, Ruin probabilities via local adjustment coefficients, J. Appl. Probab. 33 (1995), 736-755. MR.1344073 (97d:60050)

4. D. C. M. Dickson and J. R. Gray, Exact solutions for ruin probability in the presence of an absorbing upper barrier, Scand. Actuar. J. 1984, 174-186. MR781520 (86f:62178)

5. D. C. M. Dickson, The probability of ultimate ruin with a variable premium rate, Scand. Actuar. J. 1991, 75-86.

6. B. A. Djehiche, Large deviation estimate for ruin probabilities, Scand. Actuar. J. 1993, 42-59.

7. P. Embrechts and H. Schmidli, Ruin estimation for a general insurance risk model, Adv. Appl. Probab. 26 (1994), 404-422. MR 1272719 (95g:60121)

8. H. Gerber, The dilemma between dividends and safety and a generalization of the LundbergCramér formulas, Scand. Actuar. J. 1974, 46-57. MR0443874 (56:2236)

9. H. Gerber, On the probability of ruin in the presence of a linear dividend barrier, Scand. Actuar. J. 1981, 105-115. MR623405 (83c:62169)

10. H. Gerber and E. S. W. Shiu, On the time value of ruin, Proc. of the 31 Actuarial Research Conference, Ball State Univ., Aug. 1996, pp. 145-199.

11. S. S. Petersen, Calculation of ruin probabilities when the premium depends on the current reserve, Scand. Actuar. J. 1990, 147-159. MR1067100 (91f:62166)

12. P. Picard and C. Lefevre, On the first crossing of the surplus process with a given upper barrier, Insurance Math. Econom. 14 (1994), 163-179. MR1292961

13. H. Schmidli, An extension to the renewal theorem and an application to risk theory, Ann. Appl. Probab. 7 (1997), no. 1, 121-133. MR1428752 (97k:60234)

14. G. C. Tailor, Probability of ruin with variable premium rate, Scand. Actuar. J. 1980, 57-76. MR.578447 (81m:62188)

15. N. V. Kartashov, On ruin probabilities for a risk process with bounded reserves, Teor. Veroyatnost. Matem. Statist. 60 (2000), 46-58; English transl. in Theor. Probab. Math. Stat. 60 (2001), 53-65. MR1826141

16. M. V. Kartashov and O. M. Stroyev, The Lundberg approximation for the risk function in an almost homogeneous environment, Teor. Veroyatnost. Matem. Statist. 73 (2005), 63-71; English transl. in Theor. Probab. Math. Stat. 73 (2006), 71-79. MR2213842(2007b:62121)

17. N. V. Kartashov, Uniform limit theorems for ergodic stochastic processes and their application in the queueing theory, Section 1.4, Doctoral dissertation, Kiev University, Kiev, 1985. (Russian)

18. N. V. Kartashov, A generalization of Stone's representation and necessary conditions for uniform convergence in the renewal theorem, Teor. Veroyatnost. Matem. Statist. 26 (1983), 49-62; English transl. in Theor. Probab. Math. Stat. 26 (1984), 53-67. MR664903 (83m:60113)

19. N. V. Kartashov, Equivalence of uniform renewal theorems and their criteria, Teor. Veroyatnost. Matem. Statist. 27 (1983), 51-60; English transl. in Theor. Probab. Math. Stat. 27 (1984), 55-64. MR673349 (83m:60114)

20. W. Feller, An Introduction to Probability Theory and its Applications, vol. 2, John Wiley \& Sons, New York, 1966. MR0210154 (35:1048)

21. J. Grandell, Aspects of Risk Theory, Springer-Verlag, New York, 1991. MR.1084370|(92a:62151) 
22. I. I. Gikhman and A. V. Skorokhod, The Theory of Stochastic Processes, vol. 2, Nauka, Moscow, 1973; English transl., Springer-Verlag, Berlin-Heidelberg-New York, $1975 . \quad$ MR0341540 (49:6288) MR0375463 (51:11656)

23. A. A. Borovkov, Stochastic Processes in Queueing Theory, Nauka, Moscow, 1972; English transl., Springer-Verlag, New York-Heidelberg-Berlin, 1976. MR0315800 (47:4349)

24. C. Stone, On absolutely continuous components and renewal theory, Ann. Math. Stat. 37 (1966), 271-275. MR0196795 (33:4981)

25. D. Szász, Uniformity in Stone's decomposition of the renewal measure, Ann. Probab. 5 (1977), 560-564. MR0461697 (57:1681)

Department of Probability Theory and Mathematical Statistics, Faculty for Mechanics and Mathematics, National Taras Shevchenko University, Academician Glushkov Avenue 6 , KYIV 03127, UKRAINE

E-mail address: winf@ln.ua

Received 15/JAN/2007

Translated by O. I. KLESOV 